\title{
NMR-Based Metabolomics and Its Application in Drug Metabolism and Cancer Research
}

\author{
Limin Zhang ${ }^{1,2}$ • Emmanuel Hatzakis ${ }^{3}$ Andrew D. Patterson ${ }^{1}$
}

Published online: 24 August 2016

(C) Springer International Publishing AG 2016

\begin{abstract}
Purpose of Review In this review, the contribution of NMR to the metabolomics field will be discussed - particularly as it relates to cancer and drug metabolism research. An overview of the typical NMR metabolomic experiment will be presented while emphasizing important caveats ranging from experimental design to data interpretation.

Recent Findings Despite some of the drawbacks of NMR, including reduced sensitivity compared to mass spectrometry-based approaches, NMR-based metabolomic approaches have provided considerable insight into drug and cancer metabolism. Advances in flux analysis based on the strategic use of isotopes have generated unique perspectives of cancer from the cellular and tissue levels.

Summary Combined with other "omics" tools, including genomics, proteomics, and transcriptomics, metabolomic analysis completes the holistic view of metabolism. Many of these NMR-based approaches are likely to find their way into the clinic and become a common diagnostic tool.
\end{abstract}

This article is part of the Topical Collection on Pharmacometabolomics and Toxicometabolomics

Andrew D. Patterson

adp117@psu.edu

1 Center for Molecular Toxicology and Carcinogenesis, Department of Veterinary and Biomedical Sciences, The Pennsylvania State University, University Park, PA 16802, USA

2 CAS Key Laboratory of Magnetic Resonance in Biological Systems, State Key Laboratory of Magnetic Resonance and Atomic and Molecular Physics, Wuhan Centre for Magnetic Resonance, Wuhan Institute of Physics and Mathematics, Chinese Academy of Sciences (CAS), Wuhan 430071, China

3 Department of Chemistry, The Pennsylvania State University, University Park, PA 16802, USA
Keywords Metabolomics · NMR · Drug metabolism · Cancer

\section{Introduction}

Metabolomics is an informative analytical platform providing both quantitative and qualitative measurements of metabolites. Metabolomics has established itself an indispensable tool for understanding the systemic responses to developmental and/or physiological change $[1,2]$ as well as exposure to exogenous stimuli, such as diet and xenobiotics [3, 4]. Metabolomics is located downstream of genomics, transcriptomics, and proteomics and enriches the system-level view by directly assaying metabolites, their concentration, and even flux through metabolic pathways [5]. The metabolomics field continues to develop rapidly, and its exponential increase as a tool in scientific publication demonstrates its extensive application in many fields [6].

Currently, the mainstream metabolomic platforms include chromatography (e.g., liquid, gas, and capillary electrophoresis) coupled with mass spectrometry (MS), direct infusion MS, and nuclear magnetic resonance spectroscopy (NMR). MS-based metabolomic approaches in drug metabolism, biomarkers, and toxicology have been extensively reviewed elsewhere $[7,8]$. Each method has its own advantages and limitations. In reality, no perfect analytical technique can completely profile a metabolome in its entirety. The choice of suitable analytical methods therefore depends on the study objectives and the sample type to be analyzed. Employing a multiplatform approach is the best way to conduct a metabolomic investigation of complex biological samples [9]. In this review, the NMR-based metabolomic approach will be highlighted and its application in cancer and drug metabolism research. 
As a primary tool for the structural, qualitative, and quantitative analysis of biomacromolecules such as proteins and nucleic acids [10, 11], NMR-based approaches have been instrumental for the identification of metabolites (knowns and unknowns of both endogenous and xenobiotic chemicals) in biofluids (e.g., plasma, serum, and urine), cell and tissue extracts, and whole organisms. In addition to metabolite structural determination, NMR offers excellent and reproducible quantitative analysis and the potential for high throughput with flow injection technology $[12,13]$. While NMR suffers from relatively poor sensitivity compared to MS approaches, stable isotope labeling approaches (e.g., ${ }^{31} \mathrm{P},{ }^{13} \mathrm{C},{ }^{15} \mathrm{~N}$, and ${ }^{2} \mathrm{H}$ ) not only have provided structural information of unknown metabolites but also have been helpful in elucidating the flow of metabolic pathways and their connections. Furthermore, stable isotope approaches make it possible to understand and measure flux through specific pathways, which provides the metabolic rate or metabolic path as opposed to the starting and final concentration of metabolites $[14,15]$. With this information, NMR-based metabolomics has been successfully used in toxicology $[4,16,17]$, pathophysiology $[18,19]$, understanding gene function [20], and for rapid diagnosis, therapeutics, and prognosis of metabolic disease using cell, animal models, and human samples [21, 22] (Fig. 1a).

Of note, NMR-based metabolomics has also been used to identify biomarkers for cancer, including hepatocellular carcinoma (HCC) [23, 24], colon cancer [25], ovarian cancer [26], and breast cancer [27]. These approaches are essential in order to shorten the time to diagnosis - especially in cancers for which early detection and screening are difficult but would significantly impact therapeutic treatment decisions as well as prognosis. For example, Yang et al. identified metabolites in low-grade and highgrade human HCC and the adjacent normal tissue using a high-resolution, magic-angle spinning (HRMAS) ${ }^{1} \mathrm{H}$ NMR metabolomic approach [28]. Odunsi et al. used ${ }^{1} \mathrm{H}$
NMR to identify a metabolic profile or chemical fingerprint of ovarian cancer in serum by comparing results from those obtained with healthy postmenopausal women [29]. Metabolomics has also been applied in evaluating cancer radiotherapy. For example, using an isotopomer approach, Lane et al. identified a metabolite signature of apoptosis in cervical carcinoma cells following radiotherapy [15]. All of these studies demonstrate that information generated from NMR-based metabolomic studies can be useful in biomarker studies and for understanding the efficacy of treatment options.

The aims of this next section are to first describe the NMRbased metabolomic approach and to then consider which NMR platform - taking into account all advantages and disadvantages - should be considered for analysis of biofluids, tissues, and tissue extracts.

\section{NMR-Based Metabolomic Approach}

Figure $1 \mathrm{~b}$ shows the basic workflow of an NMR-based metabolomic approach. Considering the unique characteristics (e.g., editing techniques) and shortcomings (e.g., low signalto-noise and low sensitivity) of NMR, the workflow begins with experimental design, including ensuring that the study is properly powered (i.e., having appropriate sample numbers to yield informative and statistically significant results) and that confounding variables have been considered. The workflow proceeds through the biological and subsequent analytical experiments, such as sample preparation, NMR data acquisition, and signal preprocessing, on to data analysis, and then to interpretation of the results. To produce robust and valid results, it is essential to choose the correct option for specific experiments, since multiple options exist for each step in the workflow (e.g., different methods of metabolite extraction and statistical data analysis).
Fig. 1 a Schematic diagram for NMR-based metabolomic applications. b The basic workflow of NMR-based metabolomics
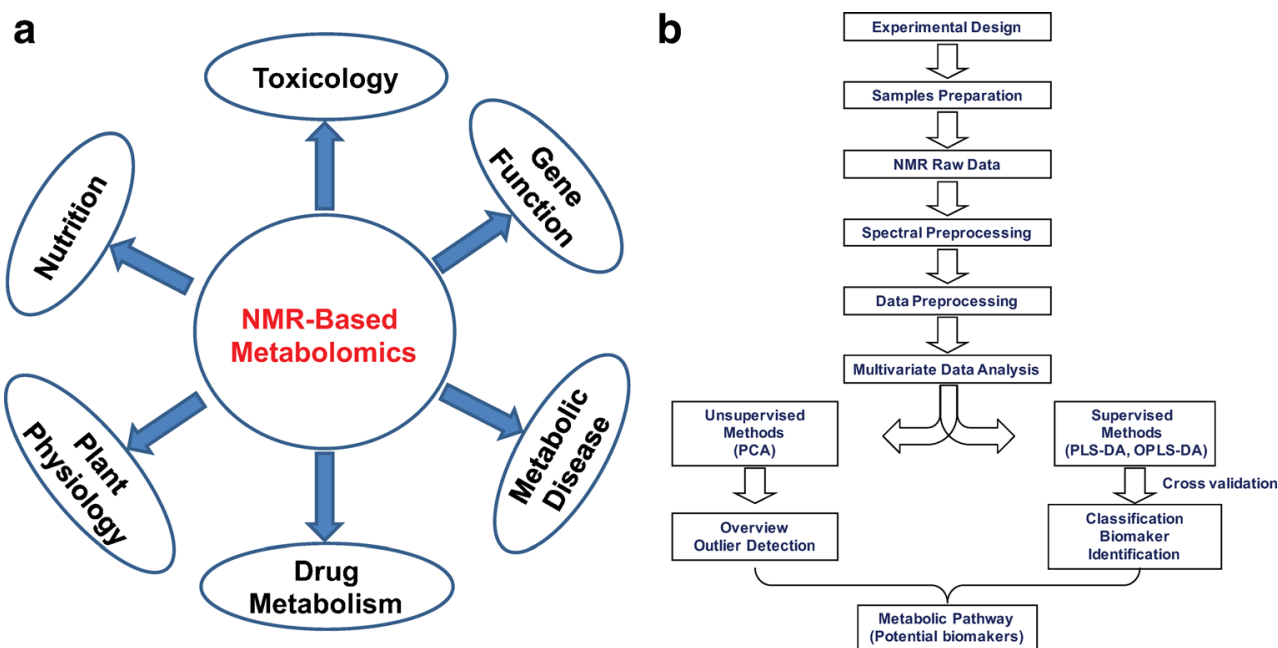
Although multiple factors influence metabolomic experiments, some can be controlled under laboratory conditions. Perhaps the most easily controlled metabolomic application is in NMR-based toxicologic research. For example, within the framework of the well-known Consortium for Metabonomics Toxicology [30, 31], which was formed by Imperial College London in collaboration with several pharmaceutical companies, an NMR-based metabolomic approach was used to develop a better understanding of organ toxicity using approximately 150 known toxicants and rodent models. Multiple factors, such as time points, dosage, and exposure pathways of toxicants, were extensively considered and controlled.

The comparisons between two or more groups, excluding potential interfering factors, are essential for extracting useful information. As for the studies related to diseases in humans, which can be difficult to adequately control due to differences in diet, lifestyle, and environment, NMR-based metabolomics has also been used to investigate the development, progression, diagnosis, and prognosis of diseases such as obesity [32] and diabetes [33], using cohorts in which groups are established as exposed (cases) versus nonexposed (controls). Within these cases, many factors, including age, gender, and lifestyle, may contribute to an observed condition, which should be considered to ensure that the two groups are as well matched as possible. In short, regardless of which NMR-based metabolomic approach is applied to highly controlled research or in clinical studies, it is critical to begin with detailed and extensively considered experimental strategy and design.

Sample requirements for metabolomics depend on the experimental goals and methods. Normally, the closer the samples' physiological status to the animal, human, or plant, the more representative the snapshot of the metabolome will be of the actual condition. For NMR-based methods, biofluids (e.g., urine, whole blood, serum, or plasma) and tissues (e.g., liver, brain, or kidney) are generally examined directly, without extensive sample preparation, so that in vivo and in situ information is easily obtained. As little as $2 \mu \mathrm{L}$ of biofluids and $10 \mathrm{mg}$ of tissues are required to obtain metabolite information with particular techniques, such as flow injection and NMR cryo probes [34-36]. For example, it is possible to analyze intact tissues (e.g., liver, kidney, and heart), plants, microbiota, or cells directly by employing HRMAS NMR to detect a chemical fingerprint $[37,38]$. This method has already been extensively applied in tissue metabolomic studies [28]. Unfortunately, some metabolites can be highly influenced by $\mathrm{pH}$ (e.g., citrate and histidine). The addition of phosphate buffer $\left(\mathrm{NaH}_{2} \mathrm{PO}_{4} /\right.$ $\left.\mathrm{K}_{2} \mathrm{HPO}_{4}\right)$ can be used to adjust the $\mathrm{pH}(\sim 7.4)$ of the solution to minimize this effect. The property of $\mathrm{pH}$ sensitivity is usually employed to investigate the microenvironment of the cellparticularly that of the cancer cell $[39,40]$. In addition, different ionic concentrations of salt in solution (especially calcium and magnesium ions in urine) can cause the chemical shift to drift, which can be optimized by adding the appropriate potassium or sodium fluoride solution to decrease the concentration of $\mathrm{Ca}^{2+}$ and $\mathrm{Mg}^{2+}$ with ion exchange reactions [41, 42]. In addition, the methods of metabolite extraction for feces and tissues in NMRbased metabolomics can be further optimized by selecting the appropriate solvents (e.g., methanol and acetonitrile) or extraction procedure, although protocols vary across research groups $[43,44]$. Samples should be snap-frozen in liquid nitrogen and stored at $-80^{\circ} \mathrm{C}$ until analysis.

After preparing samples for NMR analysis, NMR signals (e.g., those from small molecules and lipid proteins) can be obtained without separation (e.g., chromatography) of metabolites by using different NMR methods. For most biological samples, including urine and plasma/serum, aqueous extracts of feces, cells, and tissues, the typical NOESYPR1D pulse sequence (recycle delay- $90^{\circ}-t_{1}-90^{\circ}-t_{\mathrm{m}}-90^{\circ}$-acquisition) (Fig. 2a) provides solvent suppression while maintaining a flat baseline and is usually used to record a comprehensive view of the sample metabolites (Fig. 2d). However, for plasma/ serum and intact tissues containing not only a small molecule but also lipids and proteins, a water-presaturated Carr-PurcellMeiboom-Gill (CPMG) pulse sequence (recycle delay- $90^{\circ}-(\tau$ $180^{\circ}-\tau$ )n-acquisition) (Fig. 2b) and a normal $\mathrm{T}_{2}$ (spin-spin relaxation time)-edited method due to the different $\mathrm{T}_{2}$ between small molecules and macromolecules are used to attenuate NMR signals from macromolecules by adjusting the spinspin relaxation delays ( $2 \mathrm{n} \tau \sim 100 \mathrm{~ms})$ (Fig. 2e). In order to analyze the macromolecules in plasma or serum using NMR, diffusion-weighted (Fig. 2c) spectra can be acquired to uniquely distinguish NMR signals of molecules according to their diffusion coefficient (Fig. 2f). Furthermore, to improve the detection of lipids and other biomacromolecules, diffusion-ordered spectroscopy (DOSY) is commonly used to selectively remove fast-diffusing (low molecular weight) metabolites. This diffusion method is also helpful in rapidly identifying some metabolites, such as lipids and oligosaccharides, which are difficult to identify due to their significant overlap with other ${ }^{1} \mathrm{H}$ NMR signals $[45,46]$. Although many advanced NMR pulse sequences designed for structural biology research can be used to analyze the metabolome of complex systems, such as biofluids and tissues, ${ }^{1} \mathrm{H}$ NMR spectroscopy suffers from poor resolution of metabolites with similar resonances (e.g., those in the same region of the NMR spectrum), resulting again in significant overlap of signals - for instance, the thousands of ${ }^{1} \mathrm{H}$ NMR signals from all metabolites located in the spectral region of less than $10 \mathrm{ppm}$. However, 2D NMR techniques or nuclei with more dispersion, such as ${ }^{13} \mathrm{C}$ and ${ }^{31} \mathrm{P}$, can be used to help uniquely identify metabolites.

All ${ }^{1} \mathrm{H}$ NMR spectra are corrected manually for phase and baseline distortions, and spectral regions (e.g., $\delta 0.5-9.5$ for urine) are integrated into buckets with equal width (e.g., $2.4 \mathrm{~Hz}$ for $600 \mathrm{MHz}$ NMR machine). Regions distorted by imperfect water saturation and its high variability should be 
Fig. 2 The typical NOESYPR1D (a), CPMG (b), and diffusionedited (c) pulse sequences normally used in NMR-based metabolomic research and the respective spectra of plasma sample $(\mathbf{d}, \mathbf{e}, \mathbf{f})$
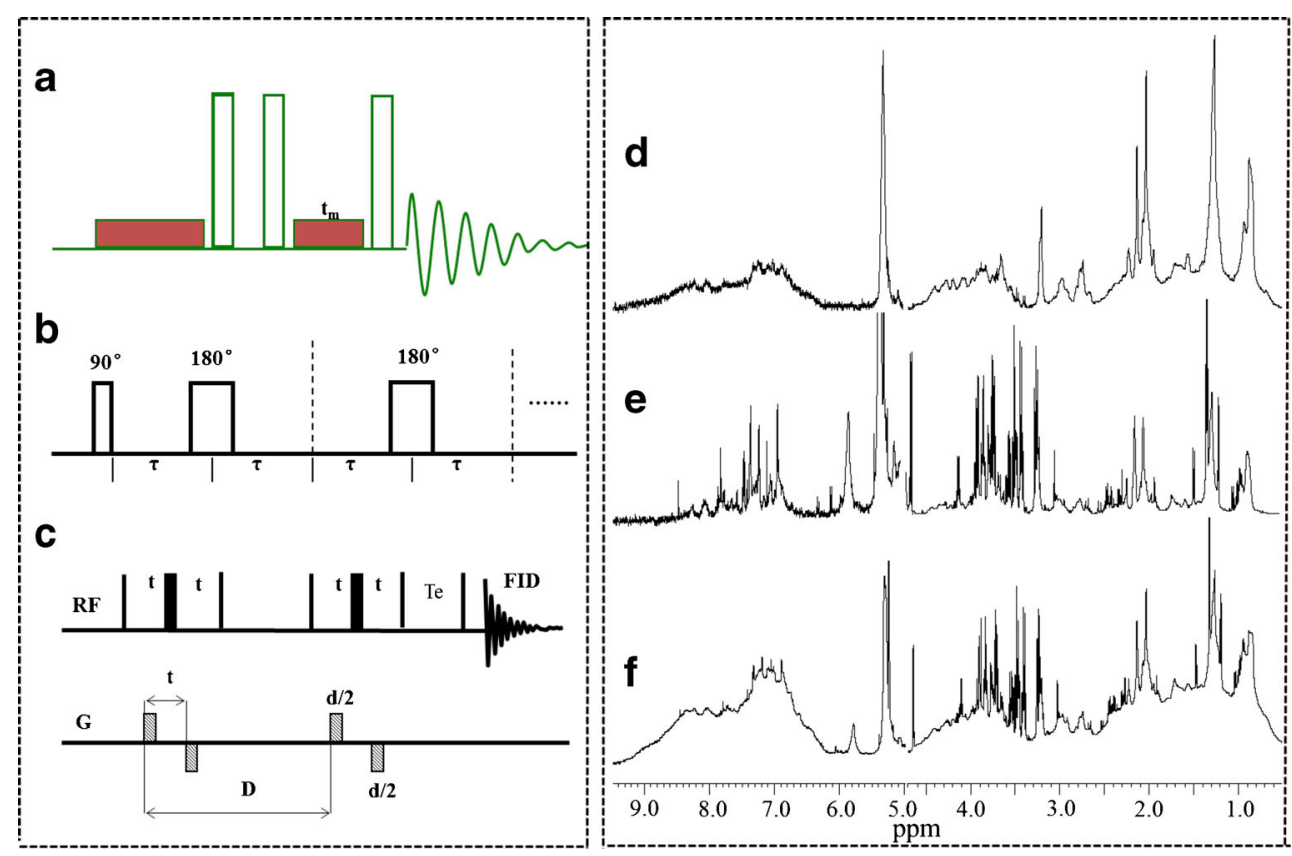

discarded with the regions containing signals of urea or exogenous compounds (e.g., contaminants including those generated during sample extraction and preparation). Each bucketed region is then normalized to compensate for the overall concentration differences prior to statistical data analysis. To facilitate NMR signal assignments, a series of 2D NMR spectra are normally employed for selected samples-including ${ }^{1} \mathrm{H}-{ }^{1} \mathrm{H}$ correlation spectroscopy (COSY), ${ }^{1} \mathrm{H}-{ }^{1} \mathrm{H}$ total correlation spectroscopy (TOCSY), ${ }^{1} \mathrm{H}-{ }^{13} \mathrm{C}$ heteronuclear singlequantum correlation (HSQC), and ${ }^{1} \mathrm{H}_{-}{ }^{13} \mathrm{C}$ heteronuclear multiple bond correlation spectra (HMBC) - and further confirmed with previous publications [47, 48] and a variety of online databases, such as the Human Metabolome Database (HMDB) [49, 50], the Madison Metabolomics Consortium Database [51], and the BioMagResBank (BMRB) [52]. As for the remaining metabolites that are difficult to identify due to low concentration, a combination of other hyphenated techniques can be used, such as liquid chromatography (LC)NMR and/or LC-NMR-MS and solid-phase extraction, which have been extensively employed in natural product studies and plant secondary metabolite identification.

To analyze NMR data, multivariate data analysis is performed to uncover latent variables that may be informative about the metabolic differences between groups. Principal component analysis (PCA), an unsupervised multivariate data analytical method, is initially employed on mean-centered NMR data to generate an overview and to identify any abnormalities or outliers within the data set. Subsequently, supervised multivariate data analytical tools, projection to latent structure discriminant analysis (PLS-DA), and orthogonal projection to latent structure discriminant analysis (OPLSDA) are applied to the analysis of ${ }^{1} \mathrm{H}$ NMR spectral data scaled to unit variance. PLS-DA and OPLS-DA models are then validated using a sevenfold cross-validation method [53] and further evaluated with a permutation test (200 permutations) and CV-ANOVA approach $(p<0.05)[54,55]$. The scores and loading plots are used to extract significantly altered metabolites with pairwise comparison.

Data visualization is preferred in metabolomic experiments due to the high dimensionality of the data collected. One particularly useful visualization method developed by the Nicholson research group is the color-coded correlation coefficient loading plot calculated from back-transformation of the loadings [4], in which the color-coded correlation coefficient indicates the significance of the metabolite contribution to the class separation, with a "hot" color (e.g., red) being more significant than a "cold" color (e.g., blue). A cutoff value is chosen according to the sample number for correlation coefficient as significant based on the discrimination significance $(p<0.05)$. Biological meaningfulness of data is the ultimate aim for metabolomic studies. Most commonly, the significantly changed metabolites obtained from multivariate statistical analysis of NMR data are mapped to a metabolic pathway according to the KEGG database [56]. However, only those relatively high-concentration metabolites are observed by the NMR technique due to its insensitivity. Many of these metabolites, such as glucose, glutamine, and adenosine triphosphate (ATP), are found in several different metabolic pathways, and they can be perturbed by a variety of diseases or other pathophysiological states. For example, the significance of glucose changes usually involved in glycolysis or gluconeogenesis is extremely different between liver or kidney toxicity and diabetes research [57]. Therefore, the interpretation of such data should be specific to the study objective 
and its interpretation carefully considered - especially as it relates to the specificity of the metabolite to the observed disease. In addition, isotope labeling $\left({ }^{13} \mathrm{C}\right.$ or $\left.{ }^{14} \mathrm{~N}\right)$ is a very powerful tool for tracking and monitoring the metabolites (usually referred to as biomarkers) to identify the specific metabolic pathway that has changed significantly-a technique that has been extensively applied in metabolic flux research [58].

\section{The Advantages and Disadvantages of NMR Spectroscopy}

NMR is an invaluable tool for chemists, structural biologists, and metabolism investigators and as such has been extensively employed in metabolic profiling research [59]. The NMRbased method is user-independent, highly reproducible, and nonselective, and it requires little or no sample preparation [60]. NMR data can provide large amounts of molecular information, including metabolite structure, concentration, and flux through pathways. Furthermore, NMR is noninvasive and nondestructive to samples, thereby permitting the re-use of the sample for other studies. For example, the recently developed HRMAS NMR method, derived from solid-state NMR technique, is carried out to achieve high-resolution NMR spectroscopy for intact tissues, which are comparable to that in the liquid state [61, 62]. In addition, signals from different metabolites can be selectively obtained by employing many NMR spectral editing techniques with pulse sequences designed according to molecular relaxation time and diffusion rate, which are very useful for spectral simplification and metabolite identification [63].

However, the polarization rate in NMR obeys the Boltzmann distribution law, resulting in small population differences between the ground and excited state of the atomic nucleus. Conventional NMR, therefore, suffers from relatively low sensitivity - the technique's major disadvantage, which is most apparent when compared with mass spectrometry. There are two ways to improve NMR sensitivity. One is to reduce electronic noise levels using the cryogenic probe technique, in which the temperature of the electronic detection circuits is reduced to approximately $20 \mathrm{~K}$, thereby increasing detection sensitivity by approximately fourfold. The second is to reduce chemical noise resulting from signal overlap using higher magnetic fields. Currently, the standard $600 \mathrm{MHz}$ spectrometer, equipped with an optimized cryogenic probe, can detect metabolites approaching nanomolar concentrations.

\section{An Overview of NMR-Based Metabolomic Application in Cancer Research}

Normal cells transforming into cancer cells usually undergo changes in cellular metabolism. During these changes, the cells consume ATP for de novo synthesis of nucleotides, lipids, and proteins, thus ensuring rapid cell proliferation.
Such molecular information of cellular metabolism plays an important role in our understanding of the development and progression of cancer. Cancer can be influenced by many factors, such as diet, environment, and lifestyle [64]. Among all of the "omics" approaches, only metabolomics technology has provided a suitable tool in investigations of pathwayspecific metabolic alterations in the development and progression of cancer and could possibly be employed in the clinical diagnosis of cancer [59]. To date, many metabolomic studies using different analytical platforms and biological matrices have been performed to identify the metabolic differences between normal cells and cancer cells [23-25]. In these studies, the NMR-based metabolomic approach provided a unique opportunity for detecting global molecular information as a readout of biochemical processes. Here, an overview of previous NMR-based metabolomic cancer studies is presented according to the sample matrices used.

\section{NMR-Based Metabolomic Analysis of Plasma/Serum and Urine in Cancer}

Blood plasma/serum NMR spectra contain signals from lipoproteins, glycoproteins, glucose, amino acids, carboxylic acids (e.g., lactate), ketone bodies, and choline metabolites. NMR-based metabolomic analysis of blood is used to generate a global overview of the whole organism metabolism. However, its composition can be influenced by different tissues, organs, and changes in the gut microbiota; therefore, changes detected in the blood may not accurately reflect the disease state. For instance, alterations in metabolites associated with glycolysis involved in energy metabolism were observed in various cancers and other diseases, such as diabetes [65]. Therefore, the interpretation of data obtained from metabolomics results should be carefully weighed not only against potentially confounding factors but also against the specificity of the biomarker identified. The well-known Warburg effect in cancer showed that glycolytic production of ATP, rather than oxidative phosphorylation via the tricarboxylic acid (TCA) cycle, is commonly observed in cancer cell metabolism [66]. The downregulation of plasma glucose, together with elevation in keto-bodies (3-hydroxybutyrate (3-HB) and pyruvate) and lactate, were also referred to as supporting evidence for cancer with a unique glucose metabolic phenotype [23]. $\mathrm{Ng}$ et al. summarized metabolic pathways and respective metabolites as biomarkers perturbed in different cancers, which indicated that some of the significantly changed metabolites can be observed in plasma or serum samples using NMR-based metabolomics [67]. In particular, those metabolites related to glucose, amino acid, and nucleic acid metabolism are commonly observed in high-resolution NMR spectra. Garcia et al. demonstrated that ${ }^{1} \mathrm{H}$ NMR-based metabolomics of serum allows for the accurate discrimination between healthy women and advanced stage III/IV and early stage I/II epithelial 
ovarian cancer (EOC) through the use of logistic regression on principal components of the NMR profiles and a statistical predictive model [26]. The observation that 3 -HB concentrations were found to have increased in both stages of EOC suggests that 3 -HB could be an early diagnostic biomarker [26]. In order to further perform targeted and cancer typespecific analysis for blood samples, isotopomer techniques combined with stable and labile metabolite extraction techniques are extensively used in NMR-based metabolomic cancer studies. For example, Fan et al. infused ${ }^{13} \mathrm{C}$ glucose, an excellent tracer molecule, into human lung cancer patients and analyzed tissues and blood plasma by ${ }^{13} \mathrm{C}$ isotopmer-based metabolomic studies using a combination of NMR and gas chromatography coupled with mass spectrometry (GC/MS) [68]. The results showed that lung tissue from lung cancer patients was identified by increased incorporation of carbon from glucose into a variety of metabolites, including TCA cycle intermediates as well as nucleotides [68]. The advantage of the stable isotope-labeled NMR metabolomic approach in cancer is the chemical library of ${ }^{13} \mathrm{C}$-labeled isotopes [69] including ${ }^{13} \mathrm{C}$-labeled amino acids (such as glutamine, serine, and glycine [70]), ${ }^{13} \mathrm{C}$-labeled acetate, and ${ }^{31} \mathrm{P}$-labeled phosphorylcholine [71] - thus allowing the determination of metabolic pathways in cancer related to protein and fatty acid synthesis, nucleic acid synthesis, nucleotide synthesis, TCA cycle, and carbohydrate metabolism. It should be noted that this approach is equally applicable to MS-based approaches.

Urine is commonly used to study bladder and kidney cancers $[72,73]$ as well as a host of other nonrenal-related studies, including hepatocellular carcinoma [74], breast cancer [75], lung cancer [76], and colorectal cancer [77]. Approximately 40 urinary endogenous metabolites can be identified from $600 \mathrm{MHz}{ }^{1} \mathrm{H}$ NMR spectra, which include amino acids, such as glycine, leucine, iosleucine, valine, alanine, and tyrosine. Also included are ketone bodies (3-HB and acetoacetate), hippurate, phenylacetylglycine (PAG), indoleacetylglycine (IAG), urea, and various organic acids, including 2oxoglutarate, citrate, taurine, succinate, fumarate, lactate, formate, $\beta$-hydroxyisovalerate, $\alpha$-hydroxyisobutyrate, and creatine. However, urine composition tends to vary with diet, and xenobiotics and their metabolites can substantially increase variability in the metabolomic profile. Usually, the concentration of those xenobiotic metabolites is too low to be detected by NMR, thereby permitting one to focus mostly on the endogenous metabolites. Among these urinary metabolites, glycine involved in methionine metabolism was observed as a biomarker in kidney cancer by ${ }^{1} \mathrm{H}$ NMR. The alterations of urinary 2-oxoglutarate, citrate, succinate, and fumarate are usually regarded as indicators of TCA cycle disruptions in almost all cases. The urinary aromatic metabolites, hippurate, PAG, and IAG are mainly produced by acyl-CoA catalysis in the liver as the conjugation of benzoic acid, phenylacetic acid, and indoleacetic acid [78]. It is noteworthy that they are also co-metabolites of host and bacteria. Therefore, the alterations of these urinary aromatic metabolites were reflective of liver dysfunction in liver cancer, and variations in their concentration may be related to modulation of activity or population of gut microbiota [79], which warrants further investigation to address the challenges of the microbiome in metabolomic research. In addition, Carrola et al. have examined the urinary metabolic profiles of lung cancer patients and controls using an NMR-based metabolomic approach [76]. The results showed that lower levels of hippurate and trigonelline, as well as higher levels of $\beta$-hydroxyisovalerate, $\alpha$-hydroxyisobutyrate, $N$ acetylglutamine, and creatinine, were observed in lung cancer patients than in controls. These putative biomarkers in urine of lung cancer were supportive evidence and were found to be specifically disease-related [76].

\section{NMR-Based Metabolomic Analysis of Intact Tissue and Tissue Extraction in Cancer}

More recently, HRMAS NMR methods have been developed to enable studies for intact tissues, which have potential to provide molecular information associated with pathology [80]. This is achieved by spinning the tissue samples at the "magic angle" $\left(54.7^{\circ}\right)$ with respect to a magnetic field so as to produce high-resolution NMR spectra from soft tissues by averaging out a range of NMR line broadening factors, such as dipole-dipole interactions, chemical shift anisotropy, and magnetic field inhomogeneity. For example, the metabolic features of human HCC in low-grade human HCC, highgrade HCC tumors, and the adjacent normal liver tissues were recently characterized employing HRMAS NMR spectroscopy in conjunction with pattern recognition techniques. This approach was useful in categorizing HCC according to histological type and grade [28]. Organ-specific biomarkers involved in metabolic pathways have also been investigated using HRMAS ${ }^{1} \mathrm{H}$ NMR-based metabolomic technique. For example, some metabolic pathways, including urea cycle (tyrosine, fumarate, arginine, and aspartate), pyrimidine metabolism (glutamine), and pyruvate metabolism (lactate and malate) were found to be related to colorectal cancer [67]. Ethanolamine and choline associated with phospholipid biosynthesis were examined as biomarkers in prostate and breast cancer tissues [67]. Griffin et al. investigated brain tumor metabolism using ${ }^{1} \mathrm{H}$ NMR-based metabolomics [81]. Furthermore, Tsang and coworkers used metabolic profiling to characterize different brain regions in rats ex vivo by HRMAS ${ }^{1} \mathrm{H}$ NMR [82]. Unique metabolite signatures were identified in the brain stem, frontal cortex, cerebellum, and hippocampus. In particular, a novel method - the combination of NMR-based metabolomics and imaging (e.g., MRI) techniques - was exploited by Cheng et al. to identify possible connections between metabolism and specific pathological conditions [83]. Using this method, $\mathrm{Wu}$ and coworkers 
evaluated prostates removed during prostatectomy from cancer patients (identified and confirmed by histology) on a $7 \mathrm{~T}$ human whole-body magnetic resonance scanner [84], and a malignancy index based on prostate cancer metabolic profiles was constructed. Furthermore, the calculation of malignancy index about the corrections between NMR metabolomic data and lesion size in cancer demonstrated a 93 to $97 \%$ overall accuracy for prostate cancer detection [84]. Such application of an NMR-based in vivo/in vitro metabolomic method suggested the great potential clinical utility of this approach.

It is conceivable that the resolution of NMR spectra obtained from tissue extraction is higher than that obtained from intact tissue. Specifically, nucleic acid signals and nucleotide metabolites are easily observed in NMR spectra of tissue extracts, whereas only a few, such as inosine and adenosine, can be detected in intact tissue due to their low concentration [85]. Therefore, purine and pyrimidine metabolism is best investigated with tissue extraction. For example, uracil and hypoxanthine were increased in colorectal, cervical, and ovarian cancers $[86,87]$, due to their need for increased DNA synthesis and cell proliferation $[86,88]$. However, the procedure and methods for tissue extraction are key factors governing the metabolomic results. Although many efforts aim to optimize the method of tissue extraction, two main shortcomings always occur: (1) the choice of solvents and extraction procedure as not all metabolites can be extracted in one method and (2) the loss of metabolites during the extraction procedure, regardless of which solvents and methods are used. The abovementioned HRMAS ${ }^{1} \mathrm{H}$ NMR method allows one to carry out experiments with intact tissues, which are much closer to the physiological status of an animal. Therefore, both intact tissues and extracts of tissues are used to perform NMRbased metabolomic research in order to ensure rigorous and reproducible results.

\section{An Overview of NMR-Based Metabolomic Application in Drug Metabolism}

Metabolite profiling and drug metabolite mapping have been informative for understanding hepatotoxicity, development toxicity, and nephrotoxicity [60, 85]. Apart from druginduced toxicity, understanding drug metabolism itself is also very important in assessing the safety and efficacy of a new drug, since drug metabolites themselves can significantly affect a drug's safety profile. Identification of biomarkers found in biofluid samples, such as blood and urine, after drug administration is expected to help elucidate the mechanism of drug action. Furthermore, drug metabolite mapping may provide important data to understand the pathophysiological effects associated with drug efficacy, selectivity, and toxicity. Therefore, in vivo animal and human studies are essential to best understand the molecular mechanism of drug action and to understand how endogenous metabolism is affected by drug metabolism.

NMR-based metabolomics is a powerful tool for systematically and rapidly identifying drug and endogenous metabolites from global profiling combined with multivariate statistical data analysis [89]. For example, NMR-based metabolic profiling was utilized to evaluate the pharmacokinetics of acetaminophen (APAP) treatment by profiling in urine its major metabolites [90]. Results indicated that the urinary level of the major APAP metabolite, $N$-acetyl- $L$-cysteine acetaminophen (APAP-NAC, a metabolite formed from the reactive intermediate $N$-acetyl-p-benzoquinone imine), was detected by NMR and found to be significantly associated with clinical chemistry data (increased ALT/AST) and endogenous oxidative stress-related metabolites such as reduced glutathione and oxidized glutathione [90]. Thus, the toxicity of APAP can be monitored indirectly through the urinary excretion of APAP-NAC and other metabolites. Interestingly, NMR-based metabolic profiling of pre-dose and post-dose urinary metabolites showed that humans with high predose levels of $p$-cresol (a metabolite often associated with gut microbiota metabolism) had lower concentrations of APAP sulfate. This observation might suggest that individuals with lower urinary concentrations of APAP sulfate might be more susceptible to APAP-induced hepatotoxicity, since less APAP was detoxified through urine by sulfate conjugation [91]. Furthermore, these observations suggest that the composition of the gut microbiota should be considered when evaluating new drugs and when studying idiosyncratic drug reactions. Previous study of the drug rosiglitazone in type 2 diabetes mellitus (T2DM) patients revealed that NMR-based metabolomic analysis could provide indications of responders, and this information should be beneficial to the clinical development of new drugs (e.g., thiazolidinediones) for T2DM [92]. Recently, Steven et al. employed NMR-based metabolomic approaches to profile the mechanisms of action of known antibiotics, such as streptomycin and ciprofloxacin, used to treat Mycobacterium tuberculosis. Results demonstrated that different classes of antibiotics uniquely modulated the metabolome of Mycobacterium smegmatis, a nonpathogenic model organism for M. tuberculosis [93, 94]. NMR-based plant metabolomics has also been employed to provide support for phytomedicine development and research, which are used extensively worldwide; study of their mechanism of action may help to identify new lead compounds [95]. Focusing on the bioactive components of herbal medicines and extracts may help to bolster claims regarding their efficacy. Taken together, these findings indicated that NMR-based metabolomics provides an efficient and unbiased approach for rapid classification, metabolism, and evaluation of lead drug candidates as well as in personalized health care. 


\section{Conclusion}

It is anticipated that NMR-based metabolomics will continue to be an important global systems biology tool and will be applied to many aspects of biomedical research, including clinical diagnosis and prognosis for cancer and other diseases. Undeniably, the combination of multiple platforms, such as NMR-based and MS-based metabolomics, and the integration and interpretation of data from various omics - including genomics, transcriptomics, and proteomics - will enable us to obtain comprehensive insight into important metabolic processes contributing to health and disease.

Acknowledgments This work was supported by National Institute of Environmental Health Sciences (A.D.P., ES022186) and the National Natural Science Foundation of China (Z.L.M., 21577169).

\section{Compliance with Ethical Standards}

Conflict of Interest The authors declare that they have no conflicts of interest.

Human and Animal Rights and Informed Consent This article does not contain any studies with human or animal subjects performed by any of the authors.

\section{References}

1. Slisz AM, Breksa 3rd AP, Mishchuk DO, McCollum G, Slupsky CM. Metabolomic analysis of citrus infection by 'Candidatus Liberibacter' reveals insight into pathogenicity. J Proteome Res. 2012;11:4223-30.

2. Kim JD, Kaiser K, Larive CK, Borkovich KA. Use of $1 \mathrm{H}$ nuclear magnetic resonance to measure intracellular metabolite levels during growth and asexual sporulation in Neurospora crassa. Eukaryot Cell. 2011;10:820-31.

3. Morgan AJ, Kille P, Sturzenbaum SR. Microevolution and ecotoxicology of metals in invertebrates. Environ Sci Technol. 2007;41: 1085-96.

4. Nicholson JK, Lindon JC, Holmes E. 'Metabonomics': understanding the metabolic responses of living systems to pathophysiological stimuli via multivariate statistical analysis of biological NMR spectroscopic data. Xenobiotica. 1999;29:1181-9.

5. D'Alessandro A, Zolla L. Meat science: from proteomics to integrated omics towards system biology. J Proteome. 2013;78:558-77.

6. Kuehnbaum NL, Britz-McKibbin P. New advances in separation science for metabolomics: resolving chemical diversity in a postgenomic era. Chem Rev. 2013;113:2437-68.

7. Johnson CH, Patterson AD, Idle JR, Gonzalez FJ. Xenobiotic metabolomics: major impact on the metabolome. Annu Rev Pharmacol Toxicol. 2012;52:37-56.

8. Patterson AD, Gonzalez FJ, Idle JR. Xenobiotic metabolism: a view through the metabolometer. Chem Res Toxicol. 2010;23:851-60.

9. Mandal R, Guo AC, Chaudhary KK, Liu P, Yallou FS, Dong E, Aziat F, et al. Multi-platform characterization of the human cerebrospinal fluid metabolome: a comprehensive and quantitative update. Genome Med. 2012;4:38.
10. Markley JL, Bax A, Arata Y, Hilbers CW, Kaptein R, Sykes BD, Wright PE, et al. Recommendations for the presentation of NMR structures of proteins and nucleic acids. IUPAC-IUBMB-IUPAB inter-union task group on the standardization of data bases of protein and nucleic acid structures determined by NMR spectroscopy. J Biomol NMR. 1998;12:1-23.

11. Davis IW, Leaver-Fay A, Chen VB, Block JN, Kapral GJ, Wang X, Murray LW, et al. MolProbity: all-atom contacts and structure validation for proteins and nucleic acids. Nucleic Acids Res. 2007;35: W375-83.

12. Da Silva L, Godejohann M, Martin FP, Collino S, Burkle A, Moreno-Villanueva M, Bernhardt J, et al. High-resolution quantitative metabolome analysis of urine by automated flow injection NMR. Anal Chem. 2013;85:5801-9.

13. Teng Q, Ekman DR, Huang W, Collette TW. Push-through direct injection NMR: an optimized automation method applied to metabolomics. Analyst. 2012;137:2226-32.

14. Godin JP, Ross AB, Rezzi S, Poussin C, Martin FP, Fuerholz A, Cleroux $\mathrm{M}$, et al. Isotopomics: a top-down systems biology approach for understanding dynamic metabolism in rats using [1,2(13)C(2)] acetate. Anal Chem. 2010;82:646-53.

15. Lane AN, Fan TW, Higashi RM. Isotopomer-based metabolomic analysis by NMR and mass spectrometry. Methods Cell Biol. 2008;84:541-88.

16. Mantle PG, Nicholls AW, Shockcor JP. H NMR spectroscopybased metabolomic assessment of uremic toxicity, with toxicological outcomes, in male rats following an acute, mid-life insult from ochratoxin a. Toxins (Basel). 2011;3:504-19.

17. Wu H, Wang WX. NMR-based metabolomic studies on the toxicological effects of cadmium and copper on green mussels Perna Viridis. Aquat Toxicol. 2010;100:339-45.

18. Basant A, Rege M, Sharma S, Sonawat HM. Alterations in urine, serum and brain metabolomic profiles exhibit sexual dimorphism during malaria disease progression. Malar J. 2010;9:110.

19. Bjerrum JT, Nielsen OH, Hao F, Tang H, Nicholson JK, Wang Y, Olsen J. Metabonomics in ulcerative colitis: diagnostics, biomarker identification, and insight into the pathophysiology. J Proteome Res. 2010;9:954-62.

20. Chen F, Zhang J, Song X, Yang J, Li H, Tang H, Liao YC. Combined metabonomic and quantitative real-time PCR analyses reveal systems metabolic changes of Fusarium graminearum induced by Tri5 gene deletion. J Proteome Res. 2011;10:2273-85.

21. Slupsky CM. NMR-based analysis of metabolites in urine provides rapid diagnosis and etiology of pneumonia. Biomark Med. 2010;4: 195-7.

22. Conti F, Manganaro M, Miccheli A. Metabolomics and medical practice. Clin Ter. 2006;157:549-52.

23. Nahon P, Amathieu R, Triba MN, Bouchemal N, Nault JC, Ziol $\mathrm{M}$, Seror $\mathrm{O}$, et al. Identification of serum proton NMR metabolomic fingerprints associated with hepatocellular carcinoma in patients with alcoholic cirrhosis. Clin Cancer Res. 2012;18:6714-22.

24. Gao H, Lu Q, Liu X, Cong H, Zhao L, Wang H, Lin D. Application of 1H NMR-based metabonomics in the study of metabolic profiling of human hepatocellular carcinoma and liver cirrhosis. Cancer Sci. 2009;100:782-5.

25. Chan EC, Koh PK, Mal M, Cheah PY, Eu KW, Backshall A, Cavill $\mathrm{R}$, et al. Metabolic profiling of human colorectal cancer using highresolution magic angle spinning nuclear magnetic resonance (HRMAS NMR) spectroscopy and gas chromatography mass spectrometry (GC/MS). J Proteome Res. 2009;8:352-61.

26. Garcia E, Andrews C, Hua J, Kim HL, Sukumaran DK, Szyperski T, Odunsi K. Diagnosis of early stage ovarian cancer by $1 \mathrm{H}$ NMR metabonomics of serum explored by use of a microflow NMR probe. J Proteome Res. 2011;10:1765-71. 
27. Yang C, Richardson AD, Smith JW, Osterman A. Comparative metabolomics of breast cancer. Pac Symp Biocomput. 2007;181-92.

28. Yang Y, Li C, Nie X, Feng X, Chen W, Yue Y, Tang H, et al. Metabonomic studies of human hepatocellular carcinoma using high-resolution magic-angle spinning $1 \mathrm{H}$ NMR spectroscopy in conjunction with multivariate data analysis. J Proteome Res. 2007;6:2605-14.

29. Odunsi K, Wollman RM, Ambrosone CB, Hutson A, McCann SE, Tammela J, Geisler JP, et al. Detection of epithelial ovarian cancer using $1 \mathrm{H}-\mathrm{NMR}$-based metabonomics. Int $\mathrm{J}$ Cancer. 2005;113:782-8.

30. Lindon JC, Nicholson JK, Holmes E, Antti H, Bollard ME, Keun $\mathrm{H}$, Beckonert $\mathrm{O}$, et al. Contemporary issues in toxicology the role of metabonomics in toxicology and its evaluation by the COMET project. Toxicol Appl Pharmacol. 2003;187:137-46.

31. Lindon JC, Keun HC, Ebbels TM, Pearce JM, Holmes E, Nicholson JK. The consortium for metabonomic toxicology (COMET): aims, activities and achievements. Pharmacogenomics. 2005;6:691-9.

32. Rasmussen LG, Winning H, Savorani F, Toft H, Larsen TM, Dragsted LO, Astrup A, et al. Assessment of the effect of high or low protein diet on the human urine metabolome as measured by NMR. Nutrients. 2012;4:112-31.

33. Griffin JL, Vidal-Puig A. Current challenges in metabolomics for diabetes research: a vital functional genomic tool or just a ploy for gaining funding? Physiol Genomics. 2008;34:1-5.

34. Xu Q, Vu H, Liu L, Wang TC, Schaefer WH. Metabolic profiles show specific mitochondrial toxicities in vitro in myotube cells. J Biomol NMR. 2011;49:207-19.

35. de Graaf RA, Chowdhury GM, Brown PB, Rothman DL, Behar KL. In situ 3D magnetic resonance metabolic imaging of microwave-irradiated rodent brain: a new tool for metabolomics research. J Neurochem. 2009;109:494-501.

36. Tang M, Zhang T, Xue Y, Wang S, Huang M, Yang Y, Lu M, et al. Dose dependent in vivo metabolic characteristics of titanium dioxide nanoparticles. J Nanosci Nanotechnol. 2010;10:8575-83.

37. Blaise BJ, Giacomotto J, Triba MN, Toulhoat P, Piotto M, Emsley $\mathrm{L}$, Segalat L, et al. Metabolic profiling strategy of Caenorhabditis elegans by whole-organism nuclear magnetic resonance. J Proteome Res. 2009;8:2542-50.

38. Bayet-Robert M, Loiseau D, Rio P, Demidem A, Barthomeuf C, Stepien G, Morvan D. Quantitative two-dimensional HRMAS 1HNMR spectroscopy-based metabolite profiling of human cancer cell lines and response to chemotherapy. Magn Reson Med. 2010;63: 1172-83.

39. Barba I, Gasparovic C, Cabanas ME, Alonso J, Murillo I, San Segundo B, Arus C. Measurement of intracellular $\mathrm{pH}$ of maize seeds (Zea mays) during germination by $31 \mathrm{P}$ nuclear magnetic resonance spectroscopy. Cell Mol Biol (Noisy-le-grand). 1997;43: 609-20.

40. Gasparovic C, Barba I, Born J, Barton S, Arus C, Mann P. A study of imidazole-based nuclear magnetic resonance probes of cellular pH. Anal Biochem. 1998;261:64-72.

41. Jiang LM, Huang J, Wang YL, Tang HR. Eliminating the dicationinduced intersample chemical-shift variations for NMR-based biofluid metabonomic analysis. Analyst. 2012;137:4209-19.

42. Xiao CN, Hao FH, Qin XR, Wang YL, Tang HR. An optimized buffer system for NMR-based urinary metabonomics with effective $\mathrm{pH}$ control, chemical shift consistency and dilution minimization. Analyst. 2009;134:916-25.

43. Wu JF, An YP, Yao JW, Wang YL, Tang HR. An optimised sample preparation method for NMR-based faecal metabonomic analysis. Analyst. 2010;135:1023-30.

44. Nicholson JK, Lindon JC. Systems biology-metabonomics. Nature. 2008;455:1054-6.
45. Kang J, Lee S, Kang S, Kwon HN, Park JH, Kwon SW, Park S. NMR-based metabolomics approach for the differentiation of ginseng (Panax ginseng) roots from different origins. Arch Pharm Res. 2008;31:330-6.

46. Tian Y, Zhang LM, Wang YL, Tang HR. Age-related topographical metabolic signatures for the rat gastrointestinal contents. J Proteome Res. 2012;11:1397-411.

47. Fan TWM, Lane AN. Structure-based profiling of metabolites and isotopomers by NMR. Prog Nucl Mag Res Spect. 2008;52:69-117.

48. Fan TWM, Lane AN, Pedler J, Crowley D, Higashi RM. Comprehensive analysis of organic ligands in whole root exudates using nuclear magnetic resonance and gas chromatography mass spectrometry. Analy Biochem. 1997;251:57-68.

49. Wishart DS, Knox C, Guo AC, Eisner R, Young N, Gautam B, Hau DD, et al. HMDB: a knowledgebase for the human metabolome. Nucleic Acids Res. 2009;37:D603-10.

50. Wishart DS, Tzur D, Knox C, Eisner R, Guo AC, Young N, Cheng D, et al. HMDB: the human metabolome database. Nucleic Acids Res. 2007;35:D521-6.

51. Markley JL, Anderson ME, Cui Q, Eghbalnia HR, Lewis IA, Hegeman AD, Li J, et al. New bioinformatics resources for metabolomics. Pac Symp Biocomput. 2007;2007:157-68.

52. Markley JL, Ulrich EL, Berman HM, Henrick K, Nakamura H, Akutsu H. BioMagResBank (BMRB) as a partner in the worldwide protein data Bank (wwPDB): new policies affecting biomolecular NMR depositions. J Biomol NMR. 2008;40:153-5.

53. Trygg J, Wold S. Orthogonal projections to latent structures $(\mathrm{O}-$ PLS). J Chemometrics. 2002;16:119-28.

54. Eriksson L, Andersson PL, Johansson E, Tysklind M. Megavariate analysis of environmental QSAR data. Part I - a basic framework founded on principal component analysis (PCA), partial least squares (PLS), and statistical molecular design (SMD). Mol Diver. 2006;10:169-86.

55. Eriksson L, Trygg J, Wold S. CV-ANOVA for significance testing of PLS and OPLS (R) models. J Chemometrics. 2008;22:594-600.

56. Okuda S, Yamada T, Hamajima M, Itoh M, Katayama T, Bork P, Goto S, et al. KEGG atlas mapping for global analysis of metabolic pathways. Nucleic Acids Res. 2008;36:W423-6.

57. Giugliano D, Ceriello A, Esposito K. Glucose metabolism and hyperglycemia. Am J Clin Nutr. 2008;87:217S-22S

58. Chikayama E, Suto M, Nishihara T, Shinozaki K, Kikuchi J. Systematic NMR analysis of stable isotope labeled metabolite mixtures in plant and animal systems: coarse grained views of metabolic pathways. PLoS One. 2008;3:e3805.

59. Reo NV. NMR-based metabolomics. Drug Chem Toxicol. 2002;25: $375-82$.

60. Nicholson JK, Connelly J, Lindon JC, Holmes E. Metabonomics: a platform for studying drug toxicity and gene function. Nat Rev Drug Discov. 2002;1:153-61.

61. Cheng LL, Chang IW, Louis DN, Gonzalez RG. Correlation of high-resolution magic angle spinning proton magnetic resonance spectroscopy with histopathology of intact human brain tumor specimens. Cancer Res. 1998;58:1825-32.

62. Bollard ME, Garrod S, Holmes E, Lindon JC, Humpfer E, Spraul $\mathrm{M}$, Nicholson JK. High-resolution (1) H and (1) H-(13)C magic angle spinning NMR spectroscopy of rat liver. Magn Reson Med. 2000;44:201-7.

63. Wang Y, Bollard ME, Keun H, Antti H, Beckonert O, Ebbels TM, Lindon JC, et al. Spectral editing and pattern recognition methods applied to high-resolution magic-angle spinning $1 \mathrm{H}$ nuclear magnetic resonance spectroscopy of liver tissues. Anal Biochem. 2003;323:26-32.

64. Matteucci E, Giampietro O. Building a bridge between clinical and basic research: the phenotypic elements of familial predisposition to type 1 diabetes. Curr Med Chem. 2007;14:555-67. 
65. Fine EJ, Segal-Isaacson CJ, Feinman RD, Herszkopf S, Romano $\mathrm{MC}$, Tomuta N, Bontempo AF, et al. Targeting insulin inhibition as a metabolic therapy in advanced cancer: a pilot safety and feasibility dietary trial in 10 patients. Nutrition. 2012;28:1028-35.

66. Pavlides S, Whitaker-Menezes D, Castello-Cros R, Flomenberg N, Witkiewicz AK, Frank PG, Casimiro MC, et al. The reverse Warburg effect: aerobic glycolysis in cancer associated fibroblasts and the tumor stroma. Cell Cycle. 2009;8:3984-4001.

67. Ng DJY, Pasikanti KK, Chan ECY. Trend analysis of metabonomics and systematic review of metabonomics-derived cancer marker metabolites. Metabolomics. 2011;7:155-78.

68. Fan TW, Lane AN, Higashi RM, Farag MA, Gao H, Bousamra M, Miller DM. Altered regulation of metabolic pathways in human lung cancer discerned by (13)C stable isotope-resolved metabolomics (SIRM). Mol Cancer. 2009;8:41.

69. Jorda J, Suarez C, Carnicer M, ten Pierick A, Heijnen JJ, van Gulik W, Ferrer P, et al. Glucose-methanol co-utilization in Pichia Pastoris studied by metabolomics and instationary (1)(3)C flux analysis. BMC Syst Biol. 2013;7:17.

70. Zhang F, Bruschweiler R. Indirect covariance NMR spectroscopy. J Am Chem Soc. 2004;126:13180-1.

71. DeSilva MA, Shanaiah N, Nagana Gowda GA, Rosa-Perez K, Hanson BA, Raftery D. Application of 31P NMR spectroscopy and chemical derivatization for metabolite profiling of lipophilic compounds in human serum. Magn Reson Chem. 2009;47(Suppl 1):S74-80.

72. Pasikanti KK, Esuvaranathan K, Ho PC, Mahendran R, Kamaraj R, $\mathrm{Wu} \mathrm{QH}$, Chiong E, et al. Noninvasive urinary metabonomic diagnosis of human bladder cancer. J Proteome Res. 2010;9:2988-95.

73. Issaq HJ, Nativ O, Waybright $T$, Luke B, Veenstra TD, Issaq EJ, Kravstov A, et al. Detection of bladder cancer in human urine by metabolomic profiling using high performance liquid chromatography/mass spectrometry. J Urol. 2008;179:2422-6.

74. Chen T, Xie G, Wang X, Fan J, Qiu Y, Zheng X, Qi X, et al. Serum and urine metabolite profiling reveals potential biomarkers of human hepatocellular carcinoma. Mol Cell Proteomics. 2011;10: M110 004945.

75. Kim Y, Koo I, Jung BH, Chung BC, Lee D. Multivariate classification of urine metabolome profiles for breast cancer diagnosis. BMC Bioinformatics. 2010;11(Suppl 2):S4.

76. Carrola J, Rocha CM, Barros AS, Gil AM, Goodfellow BJ, Carreira IM, Bernardo J, et al. Metabolic signatures of lung cancer in biofluids: NMR-based metabonomics of urine. J Proteome Res. 2011;10:221-30.

77. Qiu Y, Cai G, Su M, Chen T, Liu Y, Xu Y, Ni Y, et al. Urinary metabonomic study on colorectal cancer. J Proteome Res. 2010;9: 1627-34

78. Kasuya F, Yamaoka Y, Osawa E, Igarashi K, Fukui M. Difference of the liver and kidney in glycine conjugation of ortho-substituted benzoic acids. Chem Biol Interact. 2000;125:39-50.

79. Nicholls AW, Mortishire-Smith RJ, Nicholson JK. NMR spectroscopic-based metabonomic studies of urinary metabolite variation in acclimatizing germ-free rats. Chem Res Toxicol. 2003;16:1395-404.

80. Imperiale A, Elbayed K, Moussallieh FM, Neuville A, Piotto M, Bellocq JP, Lutz P, et al. Metabolomic pattern of childhood neuroblastoma obtained by (1)H-high-resolution magic angle spinning (HRMAS) NMR spectroscopy. Pediatr Blood Cancer. 2011;56:24-34.

81. Griffin JL, Kauppinen RA. Tumour metabolomics in animal models of human cancer. J Proteome Res. 2007;6:498-505.

82. Tsang TM, Griffin JL, Haselden J, Fish C, Holmes E. Metabolic characterization of distinct neuroanatomical regions in rats by magic angle spinning $1 \mathrm{H}$ nuclear magnetic resonance spectroscopy. Magn Reson Med. 2005;53:1018-24.

83. Spur EM, Decelle EA, Cheng LL. Metabolomic imaging of prostate cancer with magnetic resonance spectroscopy and mass spectrometry. Eur J Nucl Med Mol Imaging. 2013;40(Suppl 1):60-71.

84. Wu CL, Jordan KW, Ratai EM, Sheng JH, Adkins CB, DeFeo EM, Jenkins BG, et al. Metabolomic imaging for human prostate cancer detection. Science Transl Med. 2010;2.

85. Zhang L, Ye Y, An Y, Tian Y, Wang Y, Tang H. Systems responses of rats to aflatoxin $\mathrm{B} 1$ exposure revealed with metabonomic changes in multiple biological matrices. J Proteome Res. 2011;10:614-23.

86. Denkert C, Budczies J, Weichert W, Wohlgemuth G, Scholz M, Kind T, Niesporek $\mathrm{S}$, et al. Metabolite profiling of human colon carcinoma - deregulation of TCA cycle and amino acid turnover. Mol Cancer. 2008;7:72.

87. Denkert C, Koch I, von Keyserlingk N, Noske A, Niesporek S, Dietel M, Weichert W. Expression of the ELAV-like protein HuR in human colon cancer: association with tumor stage and cyclooxygenase-2. Mod Pathol. 2006;19:1261-9.

88. Natsumeda Y, Prajda N, Donohue JP, Glover JL, Weber G. Enzymic capacities of purine de novo and salvage pathways for nucleotide synthesis in normal and neoplastic tissues. Cancer Res. 1984;44:2475-9.

89. Azmi J, Griffin JL, Shore RF, Holmes E, Nicholson JK. Chemometric analysis of biofluids following toxicant induced hepatotoxicity: a metabonomic approach to distinguish the effects of 1naphthylisothiocyanate from its products. Xenobiotica. 2005;35: 839-52.

90. Sun J, Schnackenberg LK, Beger RD. Studies of acetaminophen and metabolites in urine and their correlations with toxicity using metabolomics. Drug Metab Lett. 2009;3:130-6.

91. Clayton TA, Baker D, Lindon JC, Everett JR, Nicholson JK. Pharmacometabonomic identification of a significant hostmicrobiome metabolic interaction affecting human drug metabolism. Proc Natl Acad Sci U S A. 2009;106:14728-33.

92. van Doorn M, Vogels J, Tas A, van Hoogdalem EJ, Burggraaf J, Cohen A, van der Greef J. Evaluation of metabolite profiles as biomarkers for the pharmacological effects of thiazolidinediones in type 2 diabetes mellitus patients and healthy volunteers. Br J Clin Pharmacol. 2007;63:562-74.

93. Steven H, Robert JF, Raúl GB, Robert P. Predicting the in vivo mechanism of action for drug leads using NMR metabolomics. ACS Chem Biol. 2012;7:166-71.

94. Stephan J, Mailaender C, Etienne G, Daffe M, Niederweis M. Multidrug resistance of a porin deletion mutant of Mycobacterium smegmatis. Antimicrob Agents Chemother. 2004;48:4163-70.

95. Shyur LF, Yang NS. Metabolomics for phytomedicine research and drug development. Curr Opin Chem Biol. 2008;12:66-71. 